\title{
Apparent reduction in the size of one side of the face associated with a small retrosplenial haemorrhage
}

\author{
Satoru Ebata, Matsuo Ogawa, Yasufumi Tanaka, Yoshikuni Mizuno, Mitsuo Yoshida
}

\begin{abstract}
A 68 year old Japanese female with unilateral metamorphopsia of the face that developed after a small haemorrhage in the contralateral retrosplenial region, is described. The patient claimed that the right side of a face, that is, the left side when looked at by the patient, appeared smaller than the left. In addition, her drawings of the face showed some distortions. Objects other than the face were perceived normally. Cranial CT scan revealed a small high density area in the right retrosplenial region. The face appears to have a special representation in the posterior hemisphere.
\end{abstract}

Metamorphopsia, which includes micropsia, macropsia and other distortions of visual images, is a rare neurological symptom and the exact location of the lesion responsible for it is not clear. It is usually a bilateral phenomenon. Our patient developed apparent reduction in size of one side of the face in her vision after a small haemorrhage in the retrosplenial region. This may have been a form of unilateral metamosphopsia.

\section{Case report}

A 60 year old right-handed Japanese house wife was apparently well until the day of admission, when she noted a peculiar sensation in her vision on awakening in the morning. When she looked into a mirror, she found the left half of her face looked smaller than the right. She was admitted to Jichi Medical School Hospital six hours after the onset.

Past medical history revealed hypertension, which had been under control for 30 years, and a right putaminal haemorrhage with residual slight loss of superficial sensation on the left side 11 years before.

On admission, she was a well nourished medium sized female in no acute distress. Her blood pressure was $170 / 110 \mathrm{~mm} \mathrm{Hg}$. General physical examination was normal. Neurological examination revealed an alert and intelligent female without any evidence of organic mental syndrome. She had no aphasia, apraxia nor agnosia. She did not have prosopagnosia. Her visual acuity and the visual field were normal. She was acutely aware that the right half of the examiner's face looked smaller than that of the left. This phenomenon was observed with the right or the left eye alone in the same way whoever she looked at. She stated that objects other than the face looked normal, and drawings of those objects had no distortion. As shown in fig 1 , her drawing of a face showed some distortions as the distance between the right eye and the centre of the face was wider despite the smaller size of the right half of the face. Extraocular muscles were all intact with normal optokinetic nystagmus. The rest of the neurological examination was entirely normal except for slight loss of superficial sensation on the left which had been present since her putaminal haemorrhage 11 years previously.

Routine blood chemistries were within normal limits. Electrocardiogram revealed sinus rhythm and negative $T$ waves in $V_{1}$ through $\mathrm{V}_{4}$. Cranial CT revealed a small high density area in the right posterior part of the cingulate gyrus just behind the splenium with a partial involvement of the adjacent corpus callosum (fig 2). Also, a small low density area was noted in each putamen. The right one most likely represents the putaminal haemorrhage 11 years before. She could not recall an
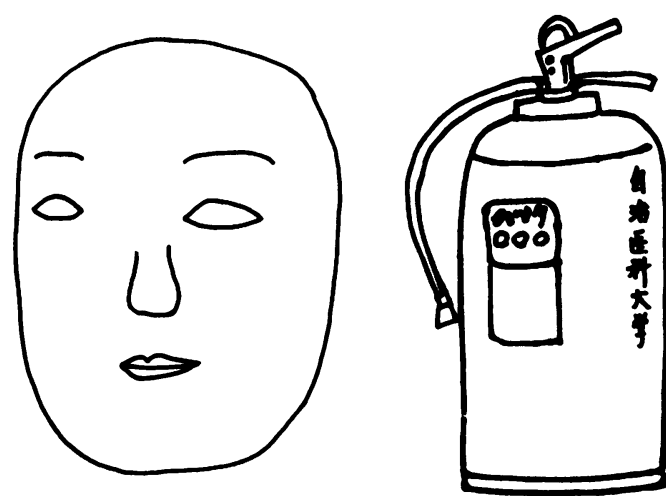

Figure 1 The patient's drawings of a face and a fire extinguisher. The right half of the face is smaller than the left, and some distortions are present in the shape of the right side of the face, in that the distance between the right eye and the centre of the face is wider than that of the left. In contrast, the fire extinguisher is drawn normally. 

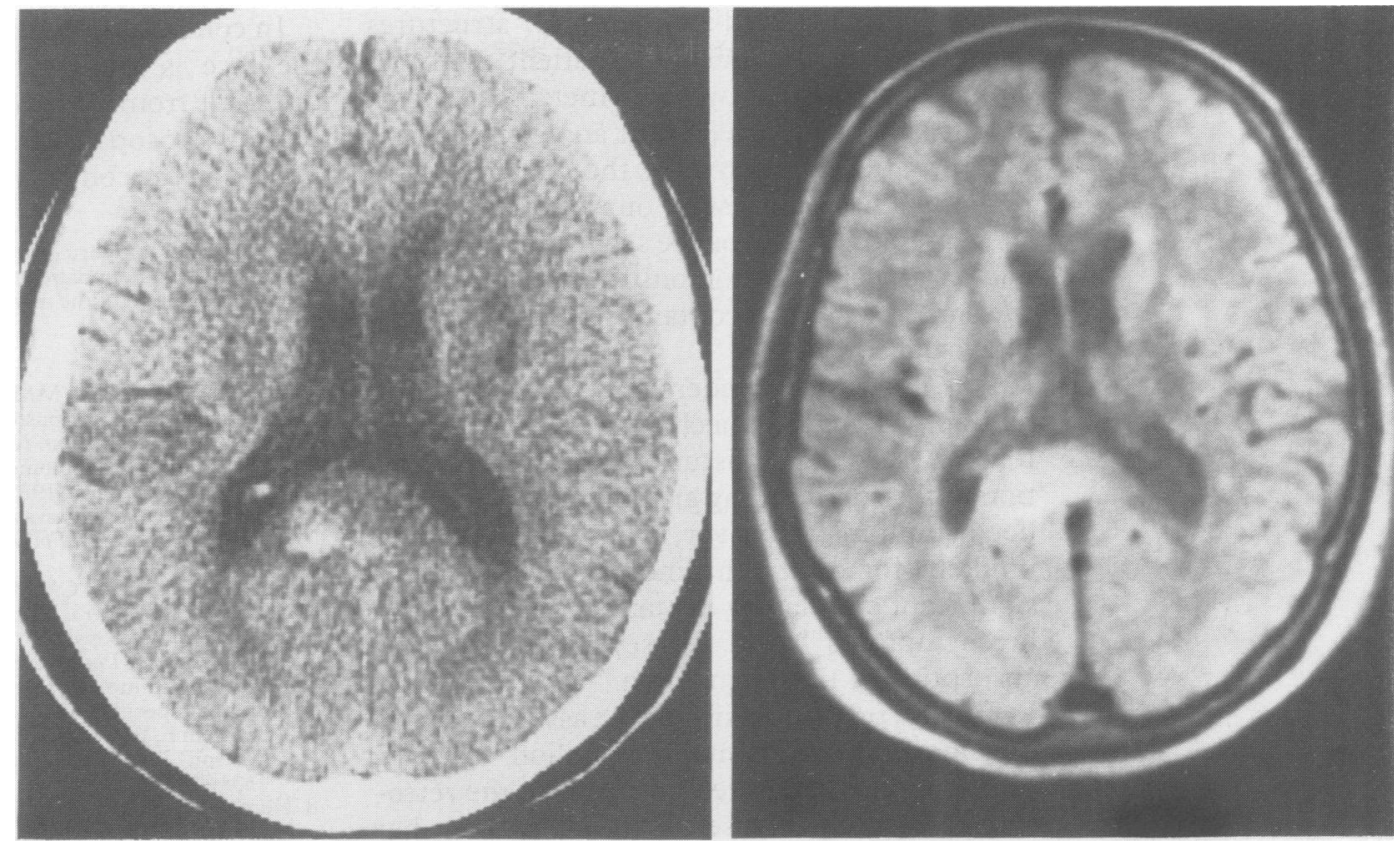

Figure 2 Left: Cranial CT images obtained at the time of admission. The right side of the brain is shown on the left side of the pictures. A small discrete high density area is seen in the right retrosplenial region with a partial involvement of the adjacent corpus callosum. Low density areas in the putamina probably represent old hemorrhages. Right: MRI obtained two weeks after the onset (proton density imaging). The right side of the brain is shown on the left side of the pictures. A small high signal area is seen involving the retrosplenial cortex extending into tr:e adjacent splenium of the corpus callosum on the right side.

episode which might have caused the low density lesion in the left putamen. Magnetic resonance imaging two weeks after the onset is also shown in fig 2 , which reveals a high signal area in the right retrosplenial region extending into the part of the adjacent splenium itself in proton density imaging. Cerebral angiography and EEG were normal.

The subsequent hospital course was uneventful, and her visual symptoms disappeared by the end of the fifth week of admission.

\section{Discussion}

Metamorphopsia including micropsia is a rare manifestation, and the anatomical substrate and the pathophysiological mechanism have not yet been well described. Interestingly her micropsia was restricted to the right half of the face (the left side as the patient looked). In addition, some distortions of the facial images were present. It seems likely therefore that she had unilateral metamorphopsia with features of micropsia. We could not be sure if her metamorphopsia was restricted to her left visual field, because we did not perform tachistoscopic examination.

Her symptoms seemed to be the result of the small haematoma in the right retrosplenial region. The nature of her haematoma was unclear. A small vascular malformation may be a possibility but we could not confirm the diagnosis and we could not find a similar case in the literature. Most of the micropsias reported were bilateral, and frequently they occurred as ictal episodes with clear epileptiform discharges in the occipital region or its vicinity. ${ }^{1-5}$

The case reported by Brust and Behrens ${ }^{1}$ is interesting in that the patient, a 44 year old diabetic man, had episodes of visual illusions in which the right half of a person's face seemed to melt, "like clocks in a Dali painting" without involvement of objects other than the face. An EEG during which he had a typical episode, was reported to have shown frequent sharp forms in the right posterior temporo-occipital area, and computerised tomography showed a right posterior temporal lucency. From this case it is clear that unilateral distortion of images restricted to the face does occur under certain circumstances. The next question is what structure may be responsible for such a phenomenon? From epileptic cases, precise localisation seems to be somewhat difficult. All the other reported epileptic micropsias were bilateral events. ${ }^{2-5}$ After reviewing those reports, all we can say is that any structure in close proximity to the occipital lobe may well be a candidate for the metamorphopsiamicropsia-inducing site.

Lance $^{6}$ reported 13 patients with visual hallucinations confined to the specific visual field defect. In six of them, the size of the objects seen in hallucinations looked smaller than the real. This is more evidence for the presence of unilateral distortions of the images. The author alluded the parieto-occipital association area to be responsible for those symptoms.

Most of the authors reporting micropsia considered the occipital visual area or the occipito-parietal or the occipito-temporal association areas as responsible for metamorphopsia including micropsia. To our knowledge, none of the reports suggested the retrosplenial region. The retrosplenial cortex is richly connected both to the anterior thalamic 
nuclei and to medial temporal structures important for memory. A patient with an amnestic syndrome (without metamosphopsia or micropsia) had bled from an arteriovenous malformation involving the retrosplenial region. ${ }^{7}$ The reason why our patient did not show memory disturbance seems to be that she had a right-sided lesion only. The splenium of the corpus callosum contains fibres connecting the visual cortices.

It is not easy to understand the underlying pathophysiological mechanism of her symptoms. A secondary disturbance of the circulation in the surrounding area might have caused her symptoms, however, it also seems rather unlikely for a tiny haemorrhage to cause a significant circulatory disturbance. In addition her symptoms lasted for several weeks after absorption of the haematoma.

Finally, prospagnosia may be associated with metamorphopsia. ${ }^{8}$ But the lesion causing prosopagnosia is located posterior to the retrosplenial region. ${ }^{9}$ It seems unlikely that her symptom was a partial phenomenon of prospagnosia.
In conclusion, unilateral metamorphopsia of the face is a distinct neurological symptom different from generalised metamorphopsia or partial prosopagnosia. The responsible site may be in the contralateral retrosplenial region.

This study was supported in part by the Research Grant from Takeda Medical Foundation. We thank Mrs Masako Chinahara for the preparation of the manuscript.

1 Brust JCM, Behrens MM. "Release hallucinations" as the major symptom of posterior cerebral artery occlusion: A report of 2 cases. Ann Neurol 1977;2:432-6.

2 Nass R, Sinha S, Solomon G. Epileptic facial metamorphopsia. Brain Dev 1985;7:50-2

3 Ida Y, Kotorii T, Nakazawa Y. A case of epilepsy with ictal metamorphopsia. Folia Psychiat Neurol Jpn 1980;34: $395-6$.

4 Russel WR, Whitty CMM. Studies in traumatic epilepsy. 3 visual fits. J Neurol Neurosurg Psychiat 1955;18:79-96.

5 Smith DL. Micropsia. An uncommon pediatric symptom. Clin Pediatr (Phila) 1980;19:297-8.

6 Lance JW. Simple formed hallucinations confined to the area of a specific visual field defect. Brain 1976;99:719-34.

Valenstein E Bowers D, Verfaellie M, Heilman KM Day A, Watson RT. Retrosplenial amnesia. Brain 1987;110: 1631-46.

8 Hecaen H, Angelergues R. Agnosia for faces (Prosopagnosia). Arch Neurol 1962;7:24-32.

9 Damasio AR, Damasio H, Hoesen WV. Prosopagnosia: Anatomic basis and behavioural mechanisms. Neurology 1982;32:331-41. 